\title{
Autoimmunity induced by human cytomegalovirus in patients with systemic lupus erythematosus
}

\author{
Cecilia Söderberg-Nauclér* \\ See related research by Hsieh et al., http://arthritis-research.com/content/13/5/R162
}

\begin{abstract}
Human cytomegalovirus is a common herpesvirus that is linked to autoimmunity, especially in genetically predisposed persons. The article by Hsieh and colleagues in a previous issue of Arthritis Research \& Therapy suggests that a C-terminal peptide of the human cytomegalovirus protein pp65 is highly immunogenic in patients with systemic lupus erythematosus and that antibodies against this peptide cross-react with nuclear proteins and double-stranded DNA, which are highly frequent autoantibodies in systemic lupus erythematosus patients. These observations highlight the fact that immunization with one small cytomegalovirus-specific peptide results in multiple autoreactive antibodies, probably through molecular mimicry and epitope spreading, in genetically predisposed persons.
\end{abstract}

In healthy people, a primary infection with human cytomegalovirus (HCMV) is often mild or even asymptomatic, but may cause a mononucleosis-like syndrome with fever, arthralgia, fatigue, thrombocytopenia, and anemia. During acute infection, HCMV infection often leads to immune dysfunction, including both immunosuppression and autoimmune phenomena. HCMV infection results in production of autoantibodies, mainly against endothelial cells and smooth muscle cells, although anti-nuclear, anti-phospholipid, and anti-CD13 autoantibodies are also common. Emerging evidence implies that HCMV can precede the onset of autoimmune disease, and this is probably more common in individuals predisposed for autoimmunity.

In a previous issue of Arthritis Research \& Therapy, Hsieh and colleagues demonstrate that a majority of

*Correspondence: cecilia.naucler@ki.se

Department of Medicine, Center for Molecular Medicine, L8:03, Karolinska Institute at Karolinska University Hospital, SE-171 76 Stockholm, Sweden systemic lupus erythematosus (SLE) patients have antibodies to the C-terminus of pp65, in particular to the subfragment $p$ 65 $5_{336-439}$ [1]. Immune reactivity against this peptide was found in 14 to $20 \%$ of patients with rheumatoid arthritis, Sjögren's syndrome, and systemic sclerosis but in only $4 \%$ of healthy controls. Immunization of BALB/c mice with pp65 $5_{336-439}$ and C3b adjuvant resulted in production of multiple antibodies that recognize nuclear structures, including chromatin, centriole mitotic spindle type I/II, and double-stranded DNA, and the immunized mice had immunoglobulin deposition in kidney glomeruli [1]. Previously, this group showed that pp65 immunization induces early autoantibody production and glomerulonephritis in lupus-prone mice. These observations suggest that the HCMV pp65 $5_{336-439}$ peptide elicits production of antibodies that cross-react with nuclear proteins and are pathogenic in genetically susceptible persons.

HCMV is a ubiquitous pathogen that infects 60 to $90 \%$ of the world's population. After a primary infection, it resides in latently infected monocytes or premonocytic cells, and reactivation often driven by inflammation may occur periodically (reviewed in [2]). Even though the virus may not trigger autoimmune disease, it may be reactivated by an initial inflammatory insult and thereafter sustain and exacerbate inflammatory processes by producing type I cytokines and by specific mechanisms that induce inflammation and autoimmune reactions. For example, HCMV infection induces expression of cyclooxygenase- 2 and 5-lipoxygenase and induces production of prostaglandin $\mathrm{E}_{2}$, leukotriene $\mathrm{B}_{4}$, and IL- 6 - all potent inflammatory mediators [3-5]. HCMV proteins alone will also drive immune reactions to nonself-peptides. By definition, autoimmune reactions occur in the absence of a pathogen in the affected organ. Emerging evidence, however, suggests that HCMV proteins are common in tissues affected by autoimmunity and that both molecular mimicry and viral antigens may sustain immune reactions.

Active HCMV infections are indeed frequent in patients with SLE, and the virus has been implicated in both development and progression of the disease (reviewed in [2]). Cytomegalovirus RNA has been detected in 
endothelial cells in skin biopsies from patients with autoimmune sclerosis, and HCMV infection is associated with higher disease activity scores in SLE patients [2]. Other autoimmune disorders linked to HCMV include vasculitis and scleroderma, rheumatoid arthritis, myositis, GuillanBarré syndrome, Wegner's granulomatosis, psoriasis, and inflammatory bowel diseases [2]. HCMV may lead to autoimmunity through molecular mimicry, epitope spreading, and an induced immune response to cryptic antigens not normally visible to the immune system.

The protein pp65 is an immunodominant T-cell epitope. During acute infection this protein generally elicits production of pp65-specific antibodies, which are apparently highly prevalent in SLE patients but poorly sustained in otherwise healthy people. Clearly, genetic susceptibility is linked to virus-induced autoimmunity and to genes encoding major histocompatibility complex and to other immune regulatory genes. For example, Toll-like receptor (TLR) 3, TLR7, and TLR9, which induce type I interferons upon ligand binding, have been associated with SLE [6]. In lupus-prone mice, TLR3, TLR7, and TLR9 signaling is required for optimal production of IgG autoantibodies and IgM rheumatoid factor [7]. Mice deficient in TLR7/9 signaling or lacking TLR3 exhibit a poor immune response to murine cytomegalovirus infection and increased mortality [8]. HCMV directly triggers plasmacytoid dendritic cells to interact with TLR7 and or TLR9 and produce IFN $\alpha$, which stimulates B cells [9]. Mutations in TLR3 increase the risk of herpes simplex virus-1 encephalitis, and carriers of a hypofunctional mutation (L412F) in the TLR3 gene have reduced activation of NF- $\mathrm{KB}$ and IFN $\gamma$ secretion, which is highly associated with severe cytomegalovirus infections, chronic candida infections, recurrent sinusitis, and hematopoietic autoimmune disorders [10].

Poor immune control of cytomegalovirus may thus lead to sustained periods of infection, and hyperfunctional TLR phenotypes may enhance the risk of autoimmunity. We followed a previously healthy woman with a primary HCMV infection who had abrupt onset of encephalitis associated with autoimmune phenomena. The patient had a TLR polymorphism and high serum levels of IFNY but extremely low levels of cytomegalovirus-specific CD4 and CD8 T cells and developed several types of autoantibodies (X Xu, P Bergman, T Willows, C Tammik; M Sund, T Hofkelt, C Soderberg-Naucler, S Varani, manuscript submitted). Prolonged treatment with ganciclovir and intravenous immunoglobulins allowed for decreasing doses of cortisone, and her neurological status improved. The findings in this case suggest that cytomegalovirus infection in subjects with potentially hidden immune defects can enhance viral replication, triggering autoimmune phenomena, and that antiviral therapy may provide beneficial effects in such individuals.
Of note, Hseih and colleagues did not observe sustained autoantibody production in mice immunized with Freund's adjuvant, but did in mice immunized with C3b [1]; and HCMV-induced autoimmunity has been described when co-infections by other pathogens were present that may enhance complement activation and the immune response [11]. In summary, HCMV may be an important trigger of autoimmunity in genetically susceptible persons, perhaps especially in patients with SLE who develop HCMV-specific antibodies that recognize nuclear structures and double-stranded DNA, which are highly prevalent in these patients. Their hyperfunctional phenotype with regards to high IFN $\alpha$ levels connects to TLR functions, which may explain the genetic susceptibility of HCMV-induced pathologies in this patient group.

\section{Abbreviations}

HCMV, human cytomegalovirus; IFN, interferon; IL, interleukin; NF, nuclear factor; SLE, systemic lupus erythematosus; TLR, Toll-like receptor.

\section{Competing interests}

The authors declare that they have no competing interests.

\section{Published: 23 January 2011}

\section{References}

1. Hsieh AH, Jhou YJ, Liang CT, Chang M, Wang SL: Fragment of tegument protein pp65 of human cytomegalovirus induces autoantibodies in BALB/c mice. Arthritis Res Ther 2011, 13:R162.

2. Varani S, Landini MP: Cytomegalovirus-induced immunopathology and its clinical consequences. Herpesviridae 2011, 2:6.

3. Qiu H, Straat K, Rahbar A, Wan M, Soderberg-Naucler C, Haeggstrom JZ: Human CMV infection induces 5-lipoxygenase expression and leukotriene B4 production in vascular smooth muscle cells. J Exp Med 2008, 205:19-24.

4. Zhu H, Cong JP, Yu D, Bresnahan WA, Shenk TE: Inhibition of cyclooxygenase 2 blocks human cytomegalovirus replication. Proc Natl Acad Sci U S A 2002, 99:3932-3937.

5. Slinger E, Maussang D, Schreiber A, Siderius M, Rahbar A, Fraile-Ramos A, Lira SA, Soderberg-Naucler C, Smit MJ: HCMV-encoded chemokine receptor US28 mediates proliferative signaling through the IL-6-STAT3 axis. Sci Signal 2010, 3:ra58

6. Baccala R, Hoebe K, Kono DH, Beutler B, Theofilopoulos AN: TLR-dependent and TLR-independent pathways of type I interferon induction in systemic autoimmunity. Nat Med 2007, 13:543-551.

7. Kono DH, Haraldsson MK, Lawson BR, Pollard KM, Koh YT, Du X, Arnold CN, Baccala R, Silverman GJ, Beutler BA, Theofilopoulos AN: Endosomal TLR signaling is required for anti-nucleic acid and rheumatoid factor autoantibodies in lupus. Proc Natl Acad Sci U S A 2009, 106:12061-12066.

8. Zucchini, N, Bessou G, Traub S, Robbins SH, Uematsu S, Akira S, Alexopoulou L, Dalod M: Cutting edge: overlapping functions of TLR7 and TLR9 for innate defense against a herpesvirus infection. J Immuno/ 2008, 180:5799-5803.

9. Varani S, Cederarv M, Feld S, Tammik C, Frascaroli G, Landini MP, SoderbergNaucler C: Human cytomegalovirus differentially controls B cell and T cell responses through effects on plasmacytoid dendritic cells. J Immuno/ 2007, 179:7767-7776.

10. Nahum A, Dadi H, Bates A, Roifman CM: The L412F variant of Toll-like receptor 3 (TLR3) is associated with cutaneous candidiasis, increased susceptibility to cytomegalovirus, and autoimmunity. J Allergy Clin Immunol 2011, 127:528-531.

11. Varani S, Mastroianni A, Frascaroli G, Tammik C, Rahbar A, Christensson M, Rossini G, Landini MP, Soderberg-Naucler C: Generalized Wegener's granulomatosis in an immunocompetent adult after cytomegalovirus mononucleosis and bacterial urinary tract infection. Arthritis Rheum 2009, 60:1558-1562.

doi:10.1186/ar3525

Cite this article as: Söderberg-Nauclér C: Autoimmunity induced by human cytomegalovirus in patients with systemic lupus erythematosus. Arthritis Research \& Therapy 2012, 14:101. 